\title{
Anti-Müllerian Hormone concentration levels in maternal plasma during the first, second and third trimester of pregnancy
}

\author{
Barbara Stegmann, MD ${ }^{1}$, Mark Santillan, MD ${ }^{1}$, Donna Santillan, Ph.D. ${ }^{1}$, Elaine \\ Smith $^{2}$ Brad Van Voorhis, MD ${ }^{1}$, Kelin Schultz, MD ${ }^{1}$
}

Keywords: Anti-Müllerian hormone, pregnancy

\section{Introduction:}

Follicle-Stimulating Hormone (FSH) drops rapidly in pregnancy but AntiMüllerian Hormone (AMH) has not been shown to drop until about 12 weeks. Since the follicles that secrete $\mathrm{AMH}$ are thought to be $\mathrm{FSH}$ independent, $\mathrm{AMH}$ levels should slowly decline in the absence of $\mathrm{FSH}$ because when the follicles reach FSH dependence, they would die off. The study by Nelson et al. presents data that suggests a decline in $\mathrm{AMH}$ levels suddenly starts at 12 weeks gestation. ${ }^{1}$

This study was performed to assess for and validate a decline of $\mathrm{AMH}$ in pregnancy and to assess for an acute versus gradual decline in $\mathrm{AMH}$ between trimesters.

${ }^{1}$ Department of Obstetrics and Gynecology, Carver College of Medicine, University of lowa Hospitals and Clinics, lowa City, IA, 52242
${ }^{2}$ Department of Epidemiology, College of Public Health, University of lowa, lowa City, IA, 52242

\section{Materials and methods:}

This retrospective cohort study was carried out on 167 samples from 112 women that were randomly distributed across all gestational ages ranging from 5.6 to 41 weeks. Fifty women provided serial samples which were accounted for in the analysis. The study was approved by the Institutional Review Board. The samples were obtained from the University of lowa (UIHC) Maternal Fetal Tissue Bank. The plasma samples were collected and then stored at -80 degrees Celsius.

The samples were then grouped based on the trimester collected; the first, second, or third trimester. $\mathrm{AMH}$ levels were then assessed for each sample using the $\mathrm{AMH}$ generation two assay kit (Beckman Coulter).

Please cite this paper as: Stegmann B, Santillan M, Santillan D, Smith E, VanVoorhis B, Schultz K. AntiMüllerian Hormone concentration levels in maternal plasma during the first, second and third trimester of pregnancy. Proc Obstet Gynecol. 2012 ;2(3):Article 2 [ 2 p.]. Available from: http://ir.uiowa.edu/pog/. Free full text article.

Corresponding author: Donna Santillan, Department of Obstetrics and Gynecology, University of lowa, 463 MRF, 200 Hawkins Drive, lowa City, IA 42242. donna-santillan@uiowa.edu. 


\section{Results:}

We observed an acute drop in $\mathrm{AMH}$ levels prior to 20 weeks of gestation when sampling women from the UIHC Maternal-Fetal Tissue Bank.

\section{Discussion:}

The present study agrees with Nelson et al. ${ }^{1}$ in regards to a decline in $\mathrm{AMH}$ after the first trimester. There is a sharp decline in $\mathrm{AMH}$ at 12-16 weeks gestation indicating that the follicular development is actively suppressed, not passively lost because of a drop in FSH. It appears that pregnancy may be a unique situation in regards to $\mathrm{AMH}$.

\section{Reference:}

${ }^{1}$ Nelson SM, Stewart F, Fleming R, Freeman DJ. Longitudinal assessment of antimüllerian hormone during pregnancyrelationship with maternal adiposity, insulin, and adiponectin. Fertil Steril. 2010 Mar 1;93(4):1356-8. 